\title{
Management of Arterial Injury in Children with Supracondylar Fracture of the Humerus and a Pulseless Hand
}

\author{
Rashid Usman, MBBS (KEMU), MRCS (UK), FCPS (Pak), ${ }^{1}$ Muhammad Jamil, MBBS, FCPS \\ (Pak), MRCS (Eng), OJT Vascular Surgery (UK), ${ }^{2}$ and Javaid Sajjad Hashmi, MBBS, FCPS \\ $(\text { Pak })^{3}$
}

\begin{abstract}
Objective: To share our experience regarding the management of arterial injuries in children with pulseless hand secondary to supracondylar fracture of the humerus (SFH).

Patients and Methods: All consecutive children with pulseless hands after SFH who were being treated in the vascular surgery units of the Combined Military Hospital, Lahore and Peshawar between September 2011 and September 2016 were included. The type of fracture, mode and pattern of injury, time from injury to definitive treatment, operative treatment, and complications were recorded.
\end{abstract}

Results: There were 55 [82\% $(n=45)$ male and $18 \%(n=10)$ female] patients with pulseless hand and SFH [Gartland type III: $18 \%(n=10)$ and IV: $82 \%(n=45)]$. The hand was cold in $38.1 \%(n=21)$ patients and warm in $61.8 \%(n=34)$. The most common mode of injury was accidental fall $[45 \%$ $(n=25)]$, and the mean time from injury to presentation was $4 \pm 2.5$ hours (mean \pm standard deviation). Overall, $71 \%$ $(n=39)$ patients underwent vascular reconstruction: autologous interposition venous grafting was performed in $49 \%$ $(n=19)$ and segmental resection and primary anastomosis in $25 \%(n=10)$ of the cases. There were no cases with amputation, fasciotomy, re-exploration, or long-term ischemic sequel.

Conclusion: Immediate vascular exploration is the treatment of choice for cold, pulseless hand. A similar approach should be adopted for warm, pulseless hand if there is no immediate return of pulse.

\footnotetext{
${ }^{1}$ Department of Vascular Surgery, Combined Military Hospital, Lahore Cantt, Pakistan

${ }^{2}$ Department of Vascular Surgery, Combined Military Hospital, Peshawar Cantt, Pakistan

${ }^{3}$ Department of Surgery, Combined Military Hospital, Lahore Cantt, Pakistan
}

Received: May 23, 2017; Accepted: August 9, 2017

Corresponding author: Rashid Usman, MBBS (KEMU), MRCS (UK), FCPS (Pak). Department of Vascular Surgery, Combined Military Hospital, 75 Sikandar block, Allama Iqbal Town, Lahore, Pakistan

Tel: +93-333-4885445, Fax: +92-423-6605551

E-mail: drrashidusman@yahoo.com
Keywords: pulseless hand, injury, brachial, supracondylar fracture, children

\section{Introduction}

Displaced supracondylar fracture of the humerus (SFH) in children is commonly associated with vascular insult in the form of absent distal pulses. ${ }^{1-3)}$ In such cases, the limb can be cold and cyanotic or warm and pink despite the loss of pulse. The vascular injury is a priority over the treatment of the fracture itself in such cases. Immediate exploration of the cold cyanotic limb is the treatment of choice for most surgeons. ${ }^{47}$ ) However, the treatment of pink, warm, pulseless limb is still under debate. Such injuries are often managed conservatively ${ }^{3-5}$; however, some researchers have also recommended early aggressive surgical intervention to prevent limb loss and serious long-term complications such as Volkmann's ischemic contracture. ${ }^{8,9)}$

Few studies have assessed the management strategies with conflicting results. Therefore, we conducted this study to review the current trends in the management of such vascular injuries associated with SFH in military tertiary care centers where microvascular experts are available at all times.

\section{Patients and Methods}

The data of all consecutive patients aged $0-16$ years with traumatic SFH who visited the emergency department of the Combined Military Hospital, Lahore and Peshawar between September 2011 and September 2016 were included in the study. All patients underwent X-ray examination of the elbow in both the anteroposterior and lateral views at the time of presentation and again after the reduction. The fractures were classified according to the Gartland classification modified by Leitch. ${ }^{10)}$

Their demographic characteristics, time between injury and presentation, type of fracture, presence or absence of 
clinical features of acute limb ischemia, type of management (conservative or operative), outcomes, and complications were recorded. Furthermore, the presence or absence of brachial, radial, and ulnar pulses; capillary refill time in the index finger pulp; and oxygen saturation using pulse oximeter were recorded in all patients.

Those patients who required surgical exploration received an intravenous bolus dose of 75 units per kilogram $(\mathrm{kg})$ of unfractioned heparin followed by continuous infusion at a rate of 25 units per $\mathrm{kg}$ per hour. Patients were operated under general anesthesia with full heparinization and antibiotic prophylaxis. Retrograde embolectomy was performed, if required. Pre-operatively, the vessels were flushed with heparinized saline. The damaged segment of the brachial artery was resected and autologous reverse venous interposition graft was placed to restore vascular continuity. Postoperatively, heparin infusion was continued at a dose of 25 units per $\mathrm{kg}$ per hour to maintain the partial thromboplastin time value at two times above its normal limit of 35-42 seconds for initial 48 hours. Anticoagulation was continued at a standard dose of subcutaneous enoxaparin ( $1 \mathrm{mg}$ per $\mathrm{kg}$ twice daily) for the next 5 days. Regular monthly follow-ups were conducted for 6 months.

Data were entered into the statistical package for social sciences version 21. Mean and standard deviation values were calculated for numerical data such as age and hospital stay. Frequency and percentage values were calculated for sex.

\section{Results}

Overall, 312 consecutive patients presented with SFH between September 2011 and September 2016. Of these, $52.8 \%(\mathrm{n}=165)$ patients had undisplaced fracture (Gartland type I) and $29.4 \%(\mathrm{n}=92)$ had sustained Gartland type II fracture. None of the patients showed clinical evidence of vascular injury; therefore, they were excluded.

In total, $17.6 \%(\mathrm{n}=55)$ patients with pulseless hand were referred to the vascular surgery department. Of them, $45.4 \%(n=25)$ had sustained the injury by falling off a motorbike/bicycle on the outstretched hand, $12.7 \%$ $(n=7)$ were hit by a moving vehicle, $27.2 \%(n=15)$ had slipped on the outstretched hand at home, and $14.5 \%$ $(\mathrm{n}=8)$ were injured while performing a sporting activity. The incidence of vascular injuries in the patients with SFH according to the modified Gartland classification is shown in Fig. 1.

In the study, $82 \%(n=45)$ of the patients were males and $18 \%(\mathrm{n}=10)$ were females, with a male to female ratio of $4.5: 1$. Left-sided injury was found in $72.7 \%$ $(\mathrm{n}=40)$, while the right brachial artery was injured in the remaining $27.3 \%(n=15)$ patients. The mean age at the

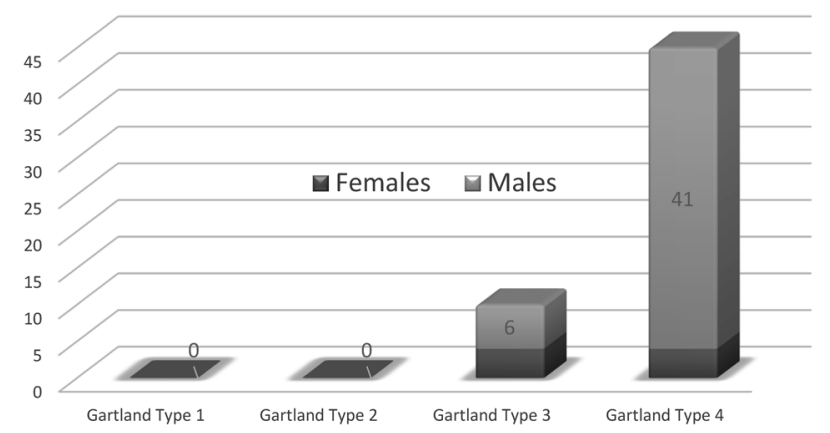

Fig. 1 Incidence of vascular injury as per the type of Gartland fracture is more appropriate.

time of presentation was $9 \pm 5$ years (mean \pm standard deviation) (range 3-16 years). The mean duration between the sustenance of injury and presentation to a vascular surgeon was $4 \pm 2.5$ hours (range $2-18$ hours). Of the total 55 cases, $83.6 \%(\mathrm{n}=46)$ presented within 6 hours, $10.9 \%$ $(\mathrm{n}=6)$ within $6-12$ hours, and $5.4 \%(\mathrm{n}=3)$ between 12 and 24 hours of the injury.

In terms of clinical presentation, radial, ulnar, and brachial pulses were absent in all cases. In the affected extremity with acute limb ischemia, pain was present in $90.9 \%(n=50)$, paresthesia in $63.6 \%(n=35)$, pallor in $54.5 \%(\mathrm{n}=30)$, coldness in $38.1 \%(\mathrm{n}=21)$, and paralysis in $5.4 \%(\mathrm{n}=3)$ patients. The patients with cold cyanotic pulseless hand $[38.1 \%(\mathrm{n}=21)]$ had a delayed capillary refill time (mean $>8$ seconds) and absent waveform and saturation on pulse oximetry. However, the patients with pink, warm, pulseless hand $[61.8 \%(\mathrm{n}=34)]$ showed a mean saturation of $85 \% \pm 3 \%$ and mean capillary refill time of $3 \pm 2$ seconds.

Of the total 34 patients with pulseless, warm hand, $56 \%(\mathrm{n}=19)$ underwent successful closed reduction and percutaneous $\mathrm{K}$ wiring with return of pulse and reversal of the acute limb ischemia signs. All these patients were scheduled to undergo a detailed duplex scan within the next 24 hours. However, in 16\% (3 out of 19) of the patients who had initially undergone a successful closed reduction, there was lose of pulse again within 6 hours of closed reduction; therefore, they underwent open exploration. The remaining $44 \%(n=15)$ patients with no pulse after closed reduction also underwent open exploration. In addition, all patients with a cold, pulseless hand [38.1\% $(n=21)]$ were surgically explored for brachial artery injury.

Of the $70.9 \%(n=39)$ patients who underwent open exploration (Figs. 2 and 3), 66.7\% $(n=26)$ had segmental contusion with thrombosis of the brachial artery. Segmental resection with end-to-end anastomosis after minimal mobilization of the ends was performed for 10 of the 26 patients. The mobilization of the ends was kept minimal to prevent any injury to the existing collateral circulation. 


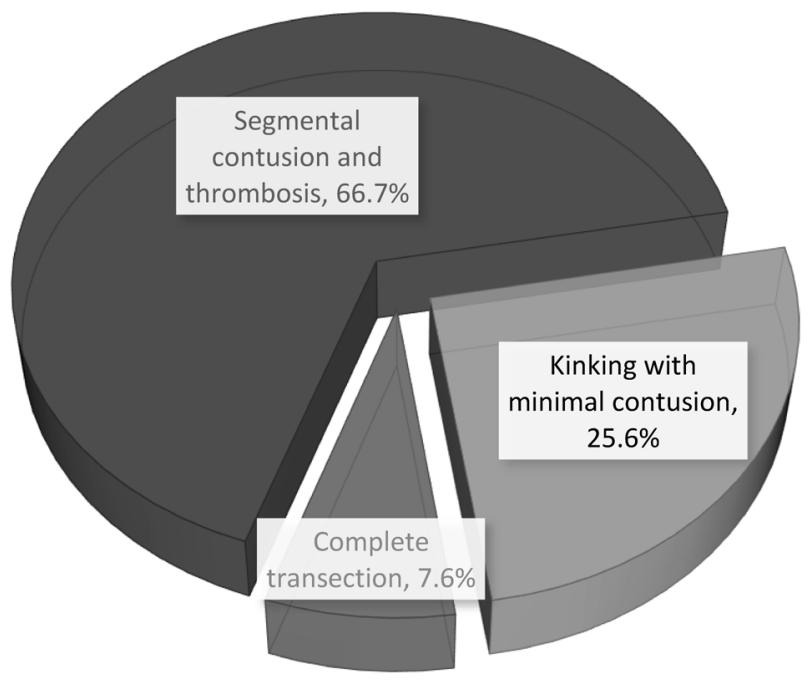

Fig. 2 Pattern of vascular injuries to the brachial artery in children with supracondylar fracture of the humerus $(n=39)$.

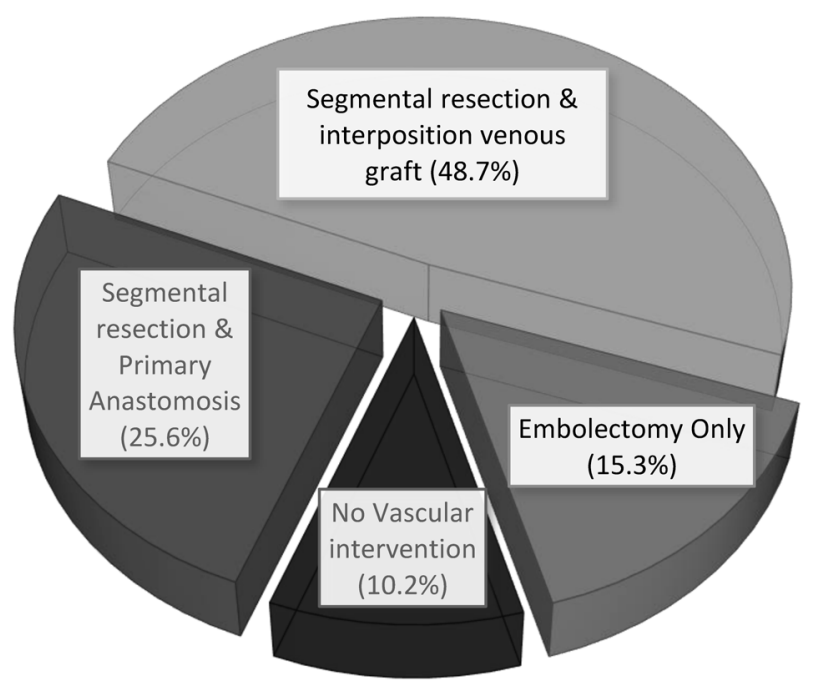

Fig. 3 Procedures performed for the arterial injuries $(n=39)$.

In 16 of the 26 patients, autologous reverse venous interposition grafting was performed because the anastomosis would have been under too much tension after the resection of the contused segment. In $25.6 \%(n=10)$ of the patients, kinking of the brachial artery was found with minimal bruising. Of these, embolectomy was indicated in 6 patients and good pulse was restored in 4 patients after bone alignment only. However, $7.6 \%(\mathrm{n}=3)$ of the patients had transection of the brachial artery with ends buried between the fractured segment. They underwent resection of the contused ends and interposition reverse venous grafting. These 3 patients also exhibited injuries to the accompanying venae comitantes that were ligated. There was no clinically demonstrable injury to the nerves in any patient.

None of the patients required a fasciotomy. The mean duration of stay in the vascular surgery department was $4 \pm 2$ days (range 2-9 days). None of the patients showed any further vascular complications during their hospital stay that warranted re-exploration. All the patients had an uneventful follow-up course for at least 6 months with no long-term ischemic sequelae such as Volkmann's ischemic contracture.

\section{Discussion}

$\mathrm{SFH}$ is a common injury in young children. It represents $16 \%$ of all pediatric fractures. ${ }^{8}$ In $97 \%$ of the cases, the fracture is caused by a fall on the outstretched hand with the elbow in full extension, causing extension type of fracture. ${ }^{11-13)}$ In the present study that involved 55 cases of SFH with vascular compromise, $87.2 \%$ patients had sustained injury while falling on the outstretched hand. The causes of such falls include slipping at home, being injured during a sporting activity, and motorbike/bicycle accidents. This type of injury is often associated with serious neurovascular damage. The documented incidence of vascular injury in such fractures is $12 \%-15 \% .{ }^{1-3)}$ The incidence of vascular trauma associated with $\mathrm{SFH}$ in our study was $17.6 \%$.

The main artery involved in SFH is the brachial artery, and the pattern of injury to the artery varies from simple contusion to laceration, thrombosis, perforation, and even transection with the ends exposed or entrapped in the fractured segments. $\left.{ }^{4}\right)$ These cases are most often diagnosed clinically. The use of additional diagnostic tools to assess circulation in a clinically pulseless limb is controversial. The handheld Doppler can be a useful adjunct to clinical examination; however, detailed duplex scan, computed tomographic angiography, magnetic resonance angiography, and conventional angiography are not recommended for diagnosing a pulseless hand. $2,4,5,11)$ Such investigations are time consuming and delay fracture repair, thereby adversely affecting the outcome. In our study involving 55 patients with pulseless hand, no patient underwent any of these investigations. The handheld Doppler probe was used in $35.5 \%(\mathrm{n}=19)$ patients to confirm the absence of pulse.

Clinically, supracondylar fractures with vascular compromise present either with a cold, pulseless cyanotic limb or with a pink, warm, pulseless limb. In our study, 38.1\% patients had cold, pulseless limb, while $61.8 \%$ had pink, warm, pulseless hand. Most previous studies recommend early intervention by a vascular surgeon for patients with cold, cyanotic, pulseless limb.4-7) All our patients with cold, cyanotic, pulseless hand underwent emergency surgical exploration, and vascular continuity was restored using microsurgical techniques. Omid et al. recommended that all patients with an absent pulse that was initially 
restored after successful closed reduction should also undergo exploration because such patients may have an entrapped brachial artery in the fracture site. $\left.{ }^{6}\right)$ Similar recommendations have been by other previous studies. ${ }^{4,5)}$ In our research, the pulse was restored after initial closed reduction, which subsequently disappeared within 6 hours of reduction in $16 \%$ of the patients. All these patients also underwent exploration of the fractured site for an assessment of the brachial artery injury.

The surgical management of pink, warm, pulseless hand in terms of vascular exploration is controversial; to date, no consensus has been established regarding its management.

Some authors believe that vascular vasospasm is intense in children and may take up to $24-48$ hours to resolve. ${ }^{3)}$ Furthermore, the chances of developing thrombosis in a kinked vessel in children are considerably lower than those in adults. ${ }^{14)}$ In addition, vascular exploration may damage the collateral circulation that may be crucial for a viable extremity. ${ }^{15)}$ In addition, complications such as reocclusion and re-stenosis following the repair of the brachial artery are common in children. ${ }^{5,16)}$ Considering all these factors, previous trials have reported that open exploration of the brachial artery is not required for cases of pulseless hand without any other symptoms. ${ }^{3-5,7,17)}$ Griffin et al. and Weller et al. recommended that bone reduction should be performed for such patients; however, it should be managed conservatively and explored surgically only if there are evident signs of ischemia. ${ }^{2,18)}$ Malviya et al. also showed a low threshold for exploration in a warm, pulseless hand. ${ }^{15)}$ In their study, the exploration rate after closed bone reduction was $16.1 \%$. Louahem and Cottalorda, in their study on 68 pink, pulseless hands, also recommended early closed reduction with closed observation and exploration only for cases with acute ischemia. ${ }^{19)}$

On the contrary, in a meta-analysis, White et al. concluded that loss of pulse in SFH is a strong indicator of brachial artery injury. ${ }^{8)}$ The collateral circulation around the elbow joint is variable in children, and success rates of brachial artery exploration are encouraging; therefore, urgent open exploration and repair is recommended. Long-term sequelae of ischemia in the forearm such as Volkmann's ischemic contracture may prove disastrous. Early aggressive management in terms of exploration of the brachial artery is recommended by Blakey et al. to prevent such serious complications. ${ }^{9)}$ Similar recommendations have been given in the POSNA questionnaire to prevent major disability. ${ }^{8)}$

It is very challenging to identify the type of injury to the brachial artery without exploration because there is a wide spectrum of possible injuries, ranging from contusion to complete transection. Some authors believe that even bone reduction should be performed under vision because blind closed reduction may further injure the already contused vessel. ${ }^{15)}$ Tunk-Naziha et al. reported only one patient who required interposition venous bypass graft; therefore, they suggested the hypothesis of open bone reduction to prevent iatrogenic vascular trauma. ${ }^{14)}$ In their study on 11 cases, Luria et al. found complete transection in $18.1 \%$ patients who underwent interposition venous grafting. ${ }^{16)}$ In our study, $7.6 \%$ patients had complete transection, and interposition venous grafting was indicated in $48.7 \%$ of the cases. Higher percentage of venous interposition graft in our patients could be the result of the cautious over-excision of the traumatized vessel that was performed considering the fact that majority of our patients were referred from other cities with a potentially poor follow-up in the future.

We did not perform a duplex scan preoperatively to identify the exact site of injury to the brachial artery; this can be considered a limitation of our study. We agree that early intervention was only possible because of the availability of microsurgical apparatus and round-theclock availability of experienced vascular surgeons. Furthermore, meticulous operative microsurgical techniques have a steep learning curve and are limited only to major centers, making it a less favorable approach for a general or orthopedic surgeon. However, our experience of early surgical intervention in a pulseless hand indicates that this is a safe approach to identify and treat vascular injuries associated with SFH in children. In addition, this approach prevents the need for a fasciotomy and lowers the chances of long-term ischemic sequelae such as Volkmann's ischemic contracture.

\section{Conclusion}

Absence of pulse in children with SFH indicates brachial artery injury even in the presence of a pink, warm hand. Immediate investigation is recommended in cases of cold, pulseless hand. Moreover, in case of pink, warm, pulseless hand, early exploration is recommended if the pulse remains absent after bony reduction. Aggressive microvascular approach must be adopted to prevent limb loss and debilitating long-term sequelae.

\section{Funding}

None.

\section{Disclosure Statement}

All authors have no conflict of interest. 


\section{Author Contributions}

Study conception: RU

Data collection: RU, JSH

Analysis: RU, MJ, JSH

Investigation: RU, JSH

Writing: RU, MJ

Critical review and revision: RU, MJ

Final approval of the article: all authors

Accountability for all aspects of the work: all authors

\section{References}

1) Garg S, Weller A, Larson N, et al. Clinical characteristics of severe supracondylar humerus fractures in children. J Pediatr Orthop 2014; 34: 34-9.

2) Griffin KJ, Walsh SR, Markar S, et al. The pink pulseless hand: a review of the literature regarding management of vascular complications of supracondylar humeral fractures in children. Eur J Vasc Endovasc Surg 2008; 36: 697-702.

3) Wegmann H, Eberl R, Kraus T, et al. The impact of arterial vessel injuries associated with pediatric supracondylar humeral fractures. J Trauma Acute Care Surg 2014; 77: 381-5.

4) Korompilias AV, Lykissas MG, Mitsionis GI, et al. Treatment of pink pulseless hand following supracondylar fractures of the humerus in children. Int Orthop 2009; 33: 237-41.

5) Erhart S, Lutz M, Larcher P, et al. A case of supracondylar humerus fracture with vascular impairment. Open it up? Arch Orthop Trauma Surg 2013; 133: 835-9.

6) Omid R, Choi D, Skaggs L. Supracondylar humeral fractures in children. J Bone Joint Surg Am 2008; 90: 1121-32.

7) Brahmamdam P, Plummer M, Modrall G, et al. Hand ischemia associated with elbow trauma in children. J Vasc Surg 2011; 54: 773-8.

8) White L, Mehlman CT, Crawford AH. Perfused, pulseless, and puzzling: a systematic review of vascular injuries in pediatric supracondylar fracture humerus fractures and results of a POSNA questionnaire. J Pediatr Orthop 2010; 30: 328-
35.

9) Blakey CM, Biant LC, Birch R. Ischemia and the pink, pulseless hand complicating supracondylar fracture of the humerus in childhood: long-term follow-up. J Bone Joint Surg Br 2009; 91: 1487-92.

10) Sinikumpu JJ, Victorzon S, Lindholm EL, et al. Ulnar nerve morbidity as a long-term complication of pediatric supracondylar humeral fracture. Musculoskelet Surg 2014; 98 : 127-33.

11) Allen SR, Hang JR, Hau RC. Review article: paediatric supracondylar humeral fractures: emergency assessment and management. Emerg Med Australas 2010; 22: 418-26.

12) Crombie A, Duncan R. Closed reduction and percutaneous fixation of displaced paediatric supracondylar fractures of the elbow. Orthop Trauma 2004; 18: 147-53.

13) Pretell-Mazzini J, Rodriguez-Martin J, Andres-Esteban M. Does open reduction and pinning affect outcome in severely displaced supracondylar humeral fractures in children? A systematic review. Strategies Trauma Limb Reconstr 2010; 5: 57-64.

14) Tunku-Naziha TZ, Wan-Yuhana W, Hadizie D, et al. Early vessels exploration of pink pulseless hand in Gartland III supracondylar fracture humerus in children: facts and controversies. Malays Orthop J 2017; 11: 12-7.

15) Malviya A, Simmons D, Vallamshetla R, et al. Pink pulseless hand following supracondylar fracture: an audit of British practice. J Pediatr Orthop B 2006; 15: 62-4.

16) Luria S, Sucar A, Eylon S, et al. Vascular complications of supracondylar humeral fractures in children. J Pediatr Orthop B 2007; 16: 133-43.

17) Ramesh P, Avadhani A, Shetty P, et al. Management of acute "pink pulseless" hand in pediatric supracondylar fractures of the humerus. J Pediatr Orthop B 2011; 20: 124-8.

18) Weller A, Garg S, Larson N, et al. Management of paediatric pulseless supracondylar humerus fracture: is vascular exploration necessary? J Bone Joint Surg Am 2013; 95: 1906-12.

19) Louahem D and Cottalorda J. Acute ischemia and pink pulseless hand in 68 of 404 Gartland type III supracondylar humeral fracture in children: urgent management and therapeutic consensus. Injury 2016; 47: 848-52. 